\title{
PROJECTIONS ONTO CONTINUOUS FUNCTION SPACES
}

\author{
D. $A M I R{ }^{1}$
}

1. Introduction. Goodner [3] introduced the family $P_{\lambda}(\lambda \geqq 1)$ of Banach spaces $X$ with the $\lambda$-projection property: For every imbedding of $X$ as a subspace of a Banach space $Z$, there exists a projection $P$ of $Z$ onto $X$ with $\|P\| \leqq \lambda$. The space $M(S)$ of all bounded real (or complex) valued functions on a set $S$, with the supremum norm, is a $P_{1}$ space, as can be seen by pointwise application of the HahnBanach theorem. $X$ is a $P_{\lambda}$ space for some finite $\lambda$ if and only if it is a direct factor in some $M(S)$ space.

A complete characterization of the $P_{1}$ spaces is known (Kelley [7], Hasumi [5]): $X$ is a $P_{1}$ space if and only if it is isometric to a space $C(S)$ of all continuous (real or complex) functions on an extremally disconnected compact Hausdorff space $S$. (A topological space is called extremally disconnected if the closure of every open set is open.) If $X$ is isomorphic to a $P_{1}$ space it is a $P_{\lambda}$ space. The open question whether these are the only $P_{\lambda}$ spaces seems to be difficult. Some necessary conditions for $X$ to be a $P_{\lambda}$ space are known. We mention the following (Grothendieck [4]): (G) If $X$ is a $P_{\lambda}$ space then every weakly* convergent sequence in $X^{*}$ is weakly convergent.

Related to the $P_{\lambda}$ spaces are the $P_{\lambda}^{\prime}$ spaces, i.e., separable Banach spaces $X$ with the separable $\lambda$-projection property: For every imbedding of $X$ as a subspace of a separable Banach space $Z$, there exists a projection $P$ of $Z$ onto $X$ with $\|P\| \leqq \lambda$. Sobczyk [10] proved that $c_{0}$ is a $P_{2}^{\prime}$ space. All known $P_{\lambda}^{\prime}$ spaces are isomorphic to $c_{0}$.

In the present paper we investigate $P_{\lambda}$ and $P_{\lambda}^{\prime}$ spaces which are continuous function spaces. $S$ will denote a compact Hausdorff space, and $C(S)$ the Banach space of all continuous real-valued functions on $S$ with the maximum norm. (With slight modifications all our results can be extended to the complex case.)

Some previous results (Isbell and Semadeni [6], Amir [1]) are:

A. If $C(S)$ is a $P_{\lambda}$ space then every convergent sequence in $S$ is eventually constant.

B. Let $i(S)$ be the maximal cardinal of families of open disjoint sets in $S$ whose closures have a nonempty intersection. If $i(S) \geqq n$

Received by the editors February 7, 1963.

${ }^{1}$ This is part of a Ph.D. thesis prepared at the Hebrew University of Jerusalem under the supervision of Professor A. Dvoretzky. 
then there exists a Banach space $Z$ such that $C(S)$ is a subspace of $Z$ (with deficiency $n-1$ ) and every projection of $Z$ onto $C(S)$ has a norm not smaller than $3-2 / n$.

Our main tool in this paper is a generalization of B (Theorem 1 ). It is used to obtain the results:

C. (Theorem 2). If $C(S)$ is a $P_{\lambda}$ space then $S$ contains a dense, open, and extremally disconnected subspace. (This theorem was obtained in [1] by a more complicated method.)

D. (Theorem 3). If $C(S)$ is a $P_{\lambda}^{\prime}$ space of an infinite dimension, then it is an isomorph of $c_{0}$.

Theorem 2 solves in the negative problem 5 in [6]: If $X$ is an infinite discrete space, then $C(\beta X-X)$ is not a $P_{\lambda}$ space (Corollary 2), although it is a continuous image of $C(\beta X)$ which is a $P_{1}$ space.

Theorem 1 enables us to construct $P_{\lambda}$ spaces of the $C(S)$ type with the exact projection constant $\lambda=1+2 \rho$ for each real $\rho$ belonging to the closure of the set

$$
\left\{\sigma ; \sigma=\sum_{i=1}^{k}\left(1-\frac{1}{n_{i}}\right) ; k, n_{i} \text { natural }\right\}
$$

(Remark c, §3).

2. Projections from $B(S, \Sigma)$ onto $C(S)$. Let $S$ be a compact Hausdorff space. Let $\Sigma$ be a field of subsets of $S$ which contains an open basis and is closed under complementation, finite union and the closure operation. $B(S, \Sigma)$ is defined as the closed subspace spanned in $M(S)$ by the characteristic functions of the sets in $\Sigma ; B(S, \Sigma)$ contains $C(S)$ as a subspace.

Definition. Let $\rho_{1}(s, \Sigma)=\sup _{n}\{1-1 / n\}$, where the supremum is taken over all $n$ such that there exist $n$ open disjoint sets $G_{1}, \cdots, G_{n}$ $\in \Sigma$ with $s \in \bigcap_{i=1}^{n} \bar{G}_{i}$.

For $k>1$ let

$$
\rho_{k}(s, \Sigma)=\sup \left\{\left(1-\frac{1}{n}\right)+\inf _{u} \min _{i=1, \cdots, n} \sup _{t \in G_{i} \cap_{u}} \rho_{k-1}(t, \Sigma)\right\},
$$

where the first supremum is taken over all finite families $\left\{G_{1}, \cdots, G_{n}\right\}$ of disjoint open sets in $\Sigma$, such that $s \in \bigcap_{i=1}^{n} \bar{G}_{i}$, and the infimum is taken over all neighbourhoods $u$ of $s$.

Let also $\rho(s, \Sigma)=\sup \left\{\rho_{k}(s, \Sigma) ; k=1,2, \cdots\right\}$ and $\rho(S, \Sigma)$ $=\sup \{\rho(s, \Sigma) ; s \in S\}$.

TheOREM 1. If $P$ is a projection of $B(S, \Sigma)$ onto $C(S)$, then $\|P\|$ $\geqq 1+2 \rho(S, \Sigma)$.

Proof. Let $\epsilon$ be an arbitrary positive number. Let $s_{1} \in S$ be such 
that $\rho(S, \Sigma) \leqq \rho\left(s_{1}, \Sigma\right)+\epsilon / 8$. We choose $k$ such that $\rho_{k}\left(s_{1}, \Sigma\right) \geqq \rho\left(s_{1}, \Sigma\right)$ $-\epsilon / 8$, and we take disjoint open sets $G(1,1), \cdots, G\left(1, n_{1}\right)$ in $\Sigma$ such that $s_{1} \in \bigcap_{i=1}^{n_{1}}[G(1, i)]^{-}$and

$$
\rho_{k}\left(s_{1}, \Sigma\right) \leqq\left(1-\frac{1}{n_{1}}\right)+\inf \min _{\imath} \sup _{t \in G(1, t) \cap_{u}} \rho_{k-1}(t, \Sigma)+\frac{\epsilon}{16 k} .
$$

Let $H(1, i)=G(1, i)$ for $i=1, \cdots, n_{1}-1$, and $H\left(1, n_{1}\right)$ $=S-\bigcup_{i=1}^{n_{1}-1} G(1, i)$.

Let $v_{1}=S, f_{1}=1$ and $X(1, i)=\chi_{H(1, i)} f_{1}$, where $\chi_{H(1, i)}$ is the characteristic function of $H(1, i)\left(i=1, \cdots, n_{1}\right)$. Let $P X(j, i ; s)$ denote the value of the function $P X(j, i)$ at the point $s$.

Since $\sum_{i=1}^{n_{1}} X(1, i)=f_{1} \in C(S)$, it follows that $\sum_{i=1}^{n_{1}} P X\left(1, i ; s_{1}\right)$ $=f_{1}\left(s_{1}\right)=1$. Hence we have for some $i_{1}$, with $1 \leqq i_{1} \leqq n_{1}, P X\left(1, i_{1} ; s_{1}\right)$ $\leqq 1 / n_{1} . P X\left(1, i_{1}\right)$ is continuous and therefore there exists a neighbourhood $u_{1}$ of $s_{1}, u_{1} \in \Sigma$, such that for every $t \in u_{1}: \mid P X\left(1, i_{1} ; t\right)$ $-P X\left(1, i_{1} ; s_{1}\right) \mid<\epsilon / 8 k$.

We proceed by induction. If $1<j<k$ and for each $r, 1 \leqq r<j$, $s_{r}, n_{r}, v_{r}, f_{r}, G(r, i), H(r, i), X(r, i)\left(1 \leqq i \leqq n_{r}\right), i_{r}$ and $u_{r}$ are defined, and satisfy the following relations:

$$
\begin{aligned}
& u_{r}, v_{r}, G(r, i), H(r, i) \in \Sigma ; \quad f_{r} \in C(S) ; \\
& s_{r} \in u_{r} \subset v_{r} \subset \bar{v}_{r} \subset u_{r-1} \cap G\left(r-1, i_{r-1}\right) ; \\
& G(r, i) \subset B(r, i) \subset G\left(r-1, i_{r-1}\right) \cap u_{r-1} ;
\end{aligned}
$$

for $1 \leqq r<j\left(\right.$ take $u_{0} \cap G\left(0, i_{0}\right)$ as $\left.S\right)$, and also:

$$
\begin{aligned}
\rho_{k-r+1}\left(s_{r}, \Sigma\right)-\rho_{k-r}\left(s_{r+1}, \Sigma\right) \leqq & \left(1-\frac{1}{n_{r}}\right)+\frac{\epsilon}{8 k} \quad \text { for } 1 \leqq r<j-1, \\
\rho_{k-j+2}\left(s_{j-1}, \Sigma\right) & \leqq\left(1-\frac{1}{n_{j-1}}\right)+\inf _{u} \min _{i} \sup _{t \in G(j-1, i) \cap u} \rho_{k-j+1}(t, \Sigma)+\frac{\epsilon}{16 k} .
\end{aligned}
$$

By the last inequality we can find $s_{j} \in u_{j-1} \cap G\left(j-1, i_{j-1}\right)$ such that $\rho_{k-j+2}\left(s_{j-1}, \Sigma\right) \leqq\left(1-\left(1 / n_{j-1}\right)\right)+\rho_{k-j+1}\left(s_{j}, \Sigma\right)+\epsilon / 8 k$. Let $v_{j} \in \Sigma$ be an open neighbourhood of $s_{j}$ such that $\bar{v}_{j} \subset u_{j-1} \cap G\left(j-1, i_{j-1}\right)$ and $f_{j} \in C(S)$ be a Urysohn function which is 1 on $v_{j}, 0$ outside $u_{j-1} \cap G\left(j-1, i_{j-1}\right)$, and $0 \leqq f_{j} \leqq 1$.

There exist $G(j, 1), \cdots, G\left(j, n_{j}\right)$ disjoint open sets in $\Sigma$ such that

$$
\rho_{k-j+1}\left(s_{j}, \Sigma\right) \leqq\left(1-\frac{1}{n_{j}}\right)+\inf _{u} \min _{i} \sup _{t \in G(j, i) \cap u} \rho_{k-j}(t, \Sigma)+\frac{\epsilon}{16 k}
$$

and we may assume that $G(j, i) \subset G\left(j-1, i_{j-1}\right) \cap u_{j-1}$ (by replacing $G$ 
by $\left.G \cap G\left(j-1, i_{j-1}\right) \cap u_{j-1}\right)$. We put $H(j, i)=G(j, i)$ for $i=1, \cdots, n_{j}-1$, and $H\left(j, n_{j}\right)=u_{j-1} \cap G\left(j-1, i_{j-1}\right)-\cup_{i=1}^{n_{i}-1} G(j, i)$.

Let $X(j, i)=\chi_{H(j, i)} f_{j}$, then $\sum_{i=1}^{n_{i}} X(j, i)=\chi_{a\left(j-1, i_{j-1}\right) \cap u_{j-1}} f_{j}=f_{j}$, and $i_{j}$ can be chosen such that $P X\left(j, i_{j} ; s_{j}\right) \leqq 1 / n_{j} . u_{j} \in \Sigma$ is an open neighbourhood of $s_{j}$ contained in $v_{j}$, in which $\left|P X\left(j, i_{j} ; t\right)-P X\left(j, i_{j} ; s_{j}\right)\right|$ $<\epsilon / 8 k$. All requirements are satisfied again, and we are ready to begin with $(j+1)$ st step.

After $k-1$ steps we choose $S_{k}$ in $u_{k-1} \cap G\left(k-1, i_{k-1}\right)$ such that

$$
\rho_{2}\left(s_{k-1}, \Sigma\right) \leqq\left(1-\frac{1}{n_{k-1}}\right)+\rho_{1}\left(s_{k}, \Sigma\right)+\frac{\epsilon}{8 k},
$$

$\boldsymbol{v}_{k}, f_{k}$ as above. $G(k, 1), \cdots, G\left(k, n_{k}\right)$ are disjoint open sets in $\Sigma$ such that $G(k, i) \subset G\left(k-1, i_{k-1}\right) \cap u_{k-1}, s_{k} \in \bigcap_{i=1}^{n_{k}}[G(K, i)]^{-}$and $\rho_{1}\left(s_{k}, \Sigma\right)$ $\leqq\left(1-\left(1 / n_{k}\right)\right)+\epsilon / 8 k . H(k, i), X(k, i)$ are defined as above and $i_{k}$ is chosen such that $P X\left(k, i_{k} ; s_{k}\right) \leqq 1 / n_{k}, u_{k} \in \Sigma$ is an open neighbourhood of $s_{k}$ contained in $v_{k}$ in which $\left|P X\left(k, i_{k} ; t\right)-P X\left(k, i_{k} ; s_{k}\right)\right|$ $<\epsilon / 8 k$.

Take an arbitrary point $s_{k+1}$ in $u_{k} \cap G\left(k, i_{k}\right)$ and a function $f_{k+1}$ $\in C(S)$ such that $f_{k+1}\left(s_{k+1}\right)=1,0 \leqq f_{k+1} \leqq 1$, and $f_{k+1}\left(S-G\left(k, i_{k}\right) \cap u_{k}\right)$ $=0$.

At last, define the function

$$
\begin{aligned}
F= & 1+2 \sum_{j=2}^{k+1} f_{j}-2 \sum_{j=1}^{k} X\left(j, i_{j}\right) \\
= & f_{1}+2 \sum_{j=2}^{k+1} f_{j}-2 \sum_{j=1}^{k} \chi_{H\left(j, i_{j}\right)} f_{j} \\
= & \left(1-2 \chi_{H\left(1, i_{1}\right)}\right) f_{1}+2 \sum_{j=2}^{k}\left(1-\chi_{H\left(j, i_{j}\right)}\right) f_{j}+2 f_{k+1} \\
= & \left(1-2 \chi_{H\left(1, i_{1}\right)}\right)\left(1-\chi_{H\left(1, i_{1}\right)}\right)+\left(1-2 \chi_{H\left(2, i_{2}\right)} f_{2}\right)\left(\chi_{H\left(1, i_{1}\right)}-\chi_{H\left(2, i_{2}\right)}\right) \\
& +\cdots+\left(1-2 \chi_{H\left(k, i_{k}\right)} f_{k}\right)\left(\chi_{H\left(k-1, i_{k-1}\right)}-\chi_{H\left(k, i_{k}\right)}\right) \\
& +\left(2 f_{k+1}-1\right) \chi_{H\left(k, i_{k}\right)}
\end{aligned}
$$

as in $H\left(j-1, i_{j-1}\right)-H\left(j, i_{j}\right), f_{r}$ is 0 for $r>j$ and 1 for $r<j$.

From the last expression it is clear that $-1 \leqq F \leqq 1$. Since the $f_{i}$ are continuous, $P F=1+2 \sum_{j=2}^{k+1} f_{j}-2 \sum_{j=1}^{k} P X\left(j, i_{j}\right)$; hence

$$
P F\left(s_{k+1}\right)=1+2 k-2 \sum_{j=1}^{k} P X\left(j, i_{j} ; s_{k+1}\right) .
$$

But $s_{k+1} \in \bigcap_{j=1}^{k} u_{j}$, hence 


$$
\begin{aligned}
\|P\| & \geqq P F\left(s_{k+1}\right) \geqq 1+2 k-2\left(\sum_{j=1}^{k} \frac{1}{n_{j}}-\frac{\epsilon}{8 k}\right) \\
& =1+2 \sum_{j=1}^{k}\left(1-\frac{1}{n_{j}}\right)-\frac{\epsilon}{4} \\
& \geqq 1+2\left\{\sum_{j=2}^{k}\left[\rho_{k-j+2}\left(s_{j-1}, \Sigma\right)-\rho_{k-j+1}\left(s_{j}, \Sigma\right)-\frac{\epsilon}{8 k}\right]\right. \\
& \left.+\rho_{1}\left(s_{k}, \Sigma\right)-\frac{\epsilon}{8 k}\right\}-\frac{\epsilon}{4} \\
& =1+2 \rho_{k}\left(s_{1}, \Sigma\right)-\frac{\epsilon}{2} \geqq 1+2 \rho\left(s_{1}, \Sigma\right)-\frac{5 \epsilon}{8} \geqq 1+2 \rho(S, \Sigma)-\epsilon .
\end{aligned}
$$

This completes the proof of Theorem 1.

Let $D_{\Sigma}$ denote the set of all boundary points of the closures of open sets in $\Sigma$, i.e., $D_{\Sigma}=\{s \in S ; s \in$ bd $\bar{G}, \Sigma \ni G$ open $\}$.

CoROLlaRY 1. If there exists a bounded projection from $B(S, \Sigma)$ onto $C(S)$, then $D_{\Sigma}$ is nowhere dense in $S$.

Proof. Suppose, on the contrary, that $D_{\Sigma}$ is dense in an open $U$. If $s \in$ bd $\bar{G}$ is an arbitrary point in $D_{\Sigma}$, then $s \in \bar{G} \cap[S-\bar{G}]^{-}$, hence $\rho_{1}(s, \Sigma) \geqq \frac{1}{2}$. By induction it follows that for every $s \in U \cap D_{\Sigma}$ we have $\rho_{k}(s, \Sigma) \geqq \frac{1}{2} k$, hence $\rho(S, \Sigma)=\infty$, which contradicts the existence of a bounded projection.

3. $P_{\lambda}$ spaces of the $C(S)$ type. Now we apply the results of the previous section to $P_{\lambda}$ spaces of the $C(S)$ type. If $\Sigma$ is the field of all subsets of $S$, we shall write $\rho(S)$ for $\rho(S, \Sigma) . B(S, \Sigma)$ in this case is simply $M(S)$. In this case Theorem 1 becomes:

Theorem $1^{\prime}$. If $C(S)$ is a $P_{\lambda}$ space, then $\lambda \geqq 1+2 \rho(S)$.

From Corollary 1 we get:

Theorem 2. If $C(S)$ is a $P_{\lambda}$ space, then $S$ contains a dense open extremally disconnected subset.

Proof. Let $D=\{s \in S: s \in$ bd $\bar{G}, G$ open $\}$. By Corollary $1, \Omega=S-\bar{D}$ is a dense subset of $S$ which is open by definition. If $G$ is an open subset of $\Omega, G$ is open in $S$ too. The closure of $G$ in $\Omega$ is contained in $\bar{G}$, hence the boundary of this closure is contained in bd $\bar{G}$ and therefore in $D$, and must be empty. This proves that $\Omega$ is also extremally disconnected. q.e.d.

Corollary 2. If $X$ is an infinite discrete space, then $C(\beta X-X)$ is not a $P_{\lambda}$ space. ( $\beta S$ denotes the Stone-Cech compactification of $S$.) 
Proof. Let $N$ be an infinite countable subset of $X . C(\beta N-N)$ is a direct factor of $C(\beta X-X)$, hence it is enough to prove that $C(\beta N-N)$ is not a $P_{\lambda}$ space. If it were a $P_{\lambda}$ space, then $\beta N-N$ would contain an open dense extremally disconnected subset, and therefore it would contain an open and closed nonvoid extremally disconnected subset of $\beta N-N$. But by a theorem of Rudin [9] an open and closed nonvoid subset of $\beta N-N$ is homeomorphic to $\beta N-N$, which is not extremally disconnected. The contradiction reached proves our assertion.

Remarks. a. $C(\beta X-X)$ is a continuous image of the $P_{1}$ space $C(\beta X)$.

b. Though the finiteness of $\rho(S)$ is a necessary condition for $C(S)$ to be a $P_{\lambda}$ space, it is not sufficient. A simple counterexample is the space $c=C\left(N^{*}\right)$ (where $N^{*}$ denotes the one-point compactification of the discrete sequence $N$ ). Since $N^{*}$ contains a convergent sequence, $C\left(N^{*}\right)$ cannot be a $P_{\lambda}$ space, although $\rho\left(N^{*}\right)=1$. A more interesting example is the following: Let $X^{\prime}$ and $X^{\prime \prime}$ be two homeomorphic discrete sets, and let $S$ be the space obtained from $\beta X^{\prime} \cup \beta X^{\prime \prime}$ by the identification of the naturally corresponding points of $\beta X^{\prime}-X^{\prime}$ and $\beta X^{\prime \prime}-X^{\prime \prime} . \rho(S)=\frac{1}{2}$, but $C(S)$ contains $c_{0}$ as a direct factor, hence is not a $P_{\lambda}$ space [6].

On the other hand, if we identify only two corresponding points: $s_{1} \in \beta X^{\prime}-X^{\prime}$ and $s_{2} \in \beta X^{\prime \prime}-X^{\prime \prime}$, we have a $P_{2}$ space with $\rho(S)=\frac{1}{2}$.

c. Starting with an infinite, extremally disconnected, compact space, and using the two procedures:

1. "Binding" a finite number of copies of a space in one point.

2. Taking the Stone-Cech compactification of the union of a family of spaces.

We can construct $S_{\rho}$ for every possible $\rho$ (i.e., belonging to the closure of $\left\{\sum_{i=1}^{k}\left(1-1 / n_{i}\right) ; k, n_{i}\right.$ natural $\}$ in the real line) such that $\rho\left(S_{\rho}\right)=\rho$ and $C\left(S_{\rho}\right)$ is a $P_{1+2 \rho}$ space (hence $1+2 \rho$ is exact).

4. $P_{\lambda}^{\prime}$ spaces of the $C(S)$ type.

THEOREM 3. The following statements are equivalent:

1. $C(S)$ is a $P_{\lambda}^{\prime}$ space for some finite $\lambda$.

2. $S$ is homeomorphic to the space of ordinals $\{\eta ; \eta \leqq \zeta\}$, with the order topology, for some $\zeta<\omega^{\omega}$.

3. $C(S)$ is isomorphic to $c_{0}$.

Proof. The implications $3 \Rightarrow 1$ and $2 \Rightarrow 3$ are simple. To prove $1 \Rightarrow 2$ we shall show first that $D=\{s \in S ; s \in$ bd $\bar{G}, G$ open $\}$ is nowhere dense in $S$. Suppose that $\bar{D}$ contains an open nonvoid $U$. Since $C(S)$ is separable, $S$ is metrizable and we can find a countable family of open sets $\left\{G_{i} ; i=1,2, \cdots\right\}$ such that $U \subset\left[\cup_{i=1}^{\infty} \text { bd } \bar{G}_{i}\right]^{-}$. 
Let $H$ be a countable open basis in $S$ which contains all the $G_{i}$, and let $\Sigma$ be the field generated by $H$ and the operations: closure, complementation, and finite union. $\Sigma$ is countable, and $B(S, \Sigma)$ is a separable Banach space containing $C(S)$, therefore there exists a bounded projection of $B(S, \Sigma)$ onto $C(S)$. By Corollary $1, D_{\Sigma}$ is nowhere dense in $S$, but this leads to contradiction since we assumed $U \subset\left[\cup_{i=1}^{\infty}\right.$ bd $\left.\bar{G}_{i}\right]-\subset D_{\Sigma}$.

$S-\bar{D}$ is an open, dense, extremally disconnected subset of $S$, and contains an open and closed, nonvoid, extremally disconnected subset. Since $S$ is metrizable, such a subset can be only finite. Hence $S$ contains an isolated point and cannot be perfect.

Next we note that if $C(S)$ is a $P_{\lambda}^{\prime}$ space and $A$ is a closed subset of $S$, then $C(A)$ is also a $P_{\lambda}^{\prime}$ space. Indeed, by a theorem of Dugundji [2], $C(A)$ is isometric to a subspace of $C(S)$ onto which there is a projection with norm 1 .

This implies that if $C(S)$ is a $P_{\lambda}^{\prime}$ space for some finite $\lambda$, then no nonvoid subset of $S$ is perfect. By a theorem of Sierpiński (Pełczyński and Semadeni [8]), $S$ is in this case homeomorphic to the space of ordinals $\{\eta ; \eta \leqq \zeta\}$ (with the order topology) for some $\zeta<\omega_{1}$.

To prove that $\zeta<\omega^{\omega}$ we note that if $\omega_{1}>\zeta \geqq \omega^{k}$, then $\rho_{k}\left(\omega^{k}, \Sigma\right)=k$, where $\Sigma$ is the countable field of subsets in $\{\eta ; \eta \leqq \zeta\}$ generated by the (countable) basis of order intervals. Applying again Theorem 1 we conclude our proof.

\section{REFERENCES}

1. D. Amir, Continuous functions' spaces with the bounded extension property, Bull. Res. Council Israel Sect. F. 10F (1962), 133-138.

2. J. Dugundji, An extension of Tietze's theorem, Pacific J. Math. 1 (1951), 353367.

3. D. B. Goodner, Projections in normed linear spaces, Trans. Amer. Math. Soc. 69 (1950), 89-108.

4. A. Grothendieck, Sur les applications linéaires faiblement compactes d'espace du type $C(K)$, Canad. J. Math. 5 (1953), 129-173.

5. M. Hasumi, The extension property of complex Banach spaces, Tôhoku Math. J. 10 (1958), 135-142.

6. J. R. Isbell and Z. Semadeni, Projection constants and spaces of continuous functions, Trans. Amer. Math. Soc. 107 (1963), 38-48.

7. J. L. Kelley, Banach spaces with the extension property, Trans. Amer. Math. Soc. 72 (1952), 323-326.

8. A. Pelczyński and Z. Semadeni, Spaces of continuous functions. III, Studia Math. 18 (1959), 211-222.

9. W. Rudin, Homogeneity problems in the theory of Čech compactification, Duke Math. J. 23 (1956), 409-419.

10. A. Sobczyk, Projection of the space $(m)$ on its subspace $\left(c_{0}\right)$, Bull. Amer. Math. Soc. 47 (1941), 938-947.

Hebrew UNIVERSity, JeRUSAlEM, IsRaEL 